\title{
Diversidade de anfíbios anuros de uma área de Pampa no município de São Gabriel, Rio Grande do Sul, Brasil
}

\author{
Ana Maria R. Bolzan ${ }^{1}$, Paulo A. Hartmann² \& Marilia T. Hartmann²
}

\footnotetext{
1. Programa de Pós-graduação em Biodiversidade Animal, Laboratório de Herpetologia, Centro de Ciências Naturais e Exatas, Universidade Federal de Santa Maria - UFSM, Av. Roraima, s/ n, Camobi, 97105-900 Santa Maria, RS, Brasil.

2. Universidade Federal da Fronteira Sul - UFFS, Campus Erechim, Av. Dom João Hoffmann, 313, 99700-000 Erechim, RS, Brasil.
}

\begin{abstract}
Diversity of anuran amphibians in an area of Pampa in the municipality of São Gabriel, Rio Grande do Sul, Brazil. Considered an endangered biome, the Pampa has one of the lowest percentages of legally protected areas. Furthermore, knowledge on anuran communities in this biome is still scarce. In this context, the aim of this study was to determine the composition of frogs in an area of Pampa in southern Brazil. Between January 2009 and February 2010 an inventory was conducted at the Fundação Estadual de Pesquisa Agropecuária in São Gabriel, Rio Grande do Sul. The search for specimens was held in permanent, semi-permanent and temporary ponds by survey method in breeding site. We recorded 21 species belonging to five families, which corresponds to approximately $20 \%$ of the species found in the state and $42 \%$ of the species cited for the Uruguayan Savanna ecoregion. Ten species were frequent, seven were considered common and four of them were considered rare. Three different reproductive modes were recorded, with $57 \%$ of the species using the first mode that appears to be related to the homogeneity of the area. Cluster analysis comparing the composition of anurans of four separate locations showed a greatest similarity with the municipality of Candiota (region of Campanha). These species are associated to grasslands in the state and neighbor countries, and might be considered typical from the Pampa biome.
\end{abstract}

KEYWORDS. Amphibia, cluster analysis, region of Campanha, community, southern fields.

RESUMO. Considerado um bioma ameaçado, o Pampa possui um dos menores percentuais de área legalmente protegida. Além disso, o conhecimento sobre comunidades de anuros neste bioma ainda é escasso. Nesse contexto, o objetivo deste estudo foi determinar a composição de anuros em uma área de Pampa no sul do Brasil. Entre janeiro de 2009 e fevereiro de 2010 foi realizado um inventário na Fundação Estadual de Pesquisa Agropecuária do município de São Gabriel, Rio Grande do Sul. A procura dos exemplares foi realizada em poças permanentes, semi-permanentes e temporárias pelo método de levantamento em sítio de reprodução. Foram registradas 21 espécies pertencentes a cinco famílias, o que corresponde a aproximadamente $20 \%$ das espécies do estado e $42 \%$ das espécies conhecidas para a ecorregião Savana Uruguaia. Dez espécies foram consideradas frequentes, sete comuns e quatro foram raras. Foram registrados três modos reprodutivos, sendo que $57 \%$ das espécies utilizam o modo 1 , que parece estar relacionado à homogeneidade da área. A análise de agrupamento comparando a composição de anuros de quatro localidades distintas mostrou maior similaridade com o município de Candiota (região da Campanha). As espécies presentes na área de estudo são associadas a formações campestres do estado e países vizinhos e podem ser consideradas típicas do bioma Pampa.

PALAVRAS-CHAVE. Amphibia, análise de agrupamento, região da Campanha, comunidade, campos sulinos.

O bioma Pampa distribui-se na porção sul da América do Sul e caracteriza-se por vegetação campestre, que predomina em relevos de planície e por vegetação mais densa, arbustiva e arbórea nas encostas e ao longo dos cursos d'água (Suertegaray \& SiLVA, 2009). A porção brasileira do bioma Pampa é encontrada apenas no estado do Rio Grande do Sul, onde resta certa de 39\% de cobertura vegetal nativa (HASENACK et al., 2007). Dentre os biomas brasileiros, é um dos que apresenta menor área legalmente protegida (IBAMA, 2006). A conservação dos campos, característicos deste bioma, sofre influência da expansão agrícola e conversão de áreas campestres em áreas destinadas à produção (SILVA, 2012). Estas transformações podem acarretar em paisagens fragmentadas, com isolamento entre habitats adequados e consequente perda de biodiversidade (SiLva, 2012).

Dentre os grupos que podem ser afetados estão os anfíbios, em função de apresentarem forte sensibilidade às alterações na estrutura da vegetação nas margens dos corpos d'água e nos parâmetros físicos e químicos da água (Duellman \& Trueb, 1994; Wells, 2007; Dodd JR., 2010). No geral, estas alterações, quando resultam de intervenções humanas, levam a um empobrecimento da estrutura e da diversidade da vegetação (LiddLE \& SCORGIE, 1980). Esta simplificação do ambiente pode causar o desaparecimento de espécies especialistas em favor de generalistas (VAN Rooy \& Stumpel, 1995). O declínio e/ou extinções de populações de anfíbios anuros provocado por modificações de habitats podem alterar a abundância e a diversidade de espécies em comunidades (Tocher et al., 1997).

A diversidade de espécies de anfíbios do Pampa ainda carece de mais informações e o conhecimento atual é considerado subestimado (BENCKE, 2009). Para o estado do Rio Grande do Sul, são conhecidas 107 espécies de anfíbios (Machado \& MALtchiK, 2007; Rosset, 2008; Iop et al., 2009; Colombo et al., 2010; CALDART et al., 2010). Para a ecorregião Savana Uruguaia, que compreende os Campos Sulinos (dos biomas Pampa e Mata Atlântica) além do Uruguai, são contabilizadas 50 espécies (GARCIA et al., 2007).

No sentido de contribuir para o conhecimento da diversidade dos anfíbios em uma área de Pampa "stricto sensu" (IBAMA, 2006), na região da Campanha Gaúcha, o este estudo objetiva estimar a riqueza de espécies na 
comunidade estudada, determinar a frequência de ocorrência das espécies encontradas, descrever os modos reprodutivos utilizados pelas espécies da comunidade e avaliar o grau de similaridade na composição da comunidade estudada com outros inventários já realizados no bioma.

\section{MATERIAL E MÉTODOS}

Área de estudo. As amostragens foram realizadas na área experimental da Fundação Estadual de Pesquisa Agropecuária - Fepagro (30²0’16,03'S; 54¹5'46,68'W, $130 \mathrm{~m}$ acima do nível do mar), município de São Gabriel, Rio Grande do Sul, Brasil (Fig. 1). A área está inserida no bioma Pampa, com formação vegetal predominantemente rasteira e arbustiva, e em menor quantidade, arbórea, caracterizado como campo graminoso e arbustivo (HASENACK et al., 2010).

Devido à posição geográfica, o sul do Brasil ocupa uma região de transição entre os climas tropical e temperado, com verões quentes e invernos frios, sem estação seca pronunciada (Overbeck et al., 2009). O clima da região é subtropical e as temperaturas médias mínimas e máximas durante o período do estudo oscilam entre $11,12^{\circ} \mathrm{C}$ e $12,41^{\circ} \mathrm{C}$ no mês mais frio, e $25,28^{\circ} \mathrm{C}$ e $26,50^{\circ} \mathrm{C}$ no mês mais quente. As chuvas são distribuídas homogeneamente durante o ano (total de $1802 \mathrm{~mm}$ no período do estudo), com as quatro estações bem definidas (PEREIRA et al., 1989).
Quatro distintos ambientes foram amostrados: Ponto 1 (30²0'30.48'S, 54¹5'40.50”W) - corpo d'água permanente e antropizado (açude), com 147,15 $\mathrm{m}^{2}$ de área (calculado pela fórmula da elipse), e predomínio de gramíneas na vegetação marginal. Ponto 2 (30²0’28.12”S, $\left.54^{\circ} 15^{\prime} 35.76^{\prime} \mathrm{W}\right)$ - poça semipermanente, com $1613,16 \mathrm{~m}^{2}$ de área. Em períodos longos sem chuva quase seca. Ponto

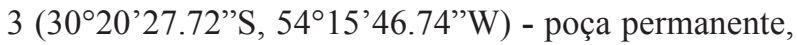
com $1951,47 \mathrm{~m}^{2}$ de área. Apresenta predomínio de gramíneas no interior e entorno. Ponto 4 (30²0’20.95'S, $\left.54^{\circ} 15^{\prime} 31.52^{\prime \prime} \mathrm{W}\right)$ - poça temporária, com $575,88 \mathrm{~m}^{2}$ de área, acumula água somente após grande volume de chuvas. A vegetação de entorno é composta por gramíneas, caraguatá e algumas árvores.

Coleta de dados. $\mathrm{O}$ estudo foi realizado de janeiro de 2009 a fevereiro de 2010, com exceção dos meses de fevereiro e julho de 2009 que, por questões logísticas, não puderam ser amostrados. Foram realizadas amostras em três dias por mês utilizando o método de "busca em sítio de reprodução" (SCOTT JR. \& WoOdWARD, 1994). As observações ocorreram entre 18:00 e 23:00 horas e foram conduzidas por três pessoas, totalizando 495 horas de esforço amostral.

Complementarmente foi efetuada procura ativa por espécies durante o dia, por pelo menos uma hora/mês. Essa metodologia consiste na procura de anfíbios em seus possíveis refúgios, como embaixo de troncos e pedras, no

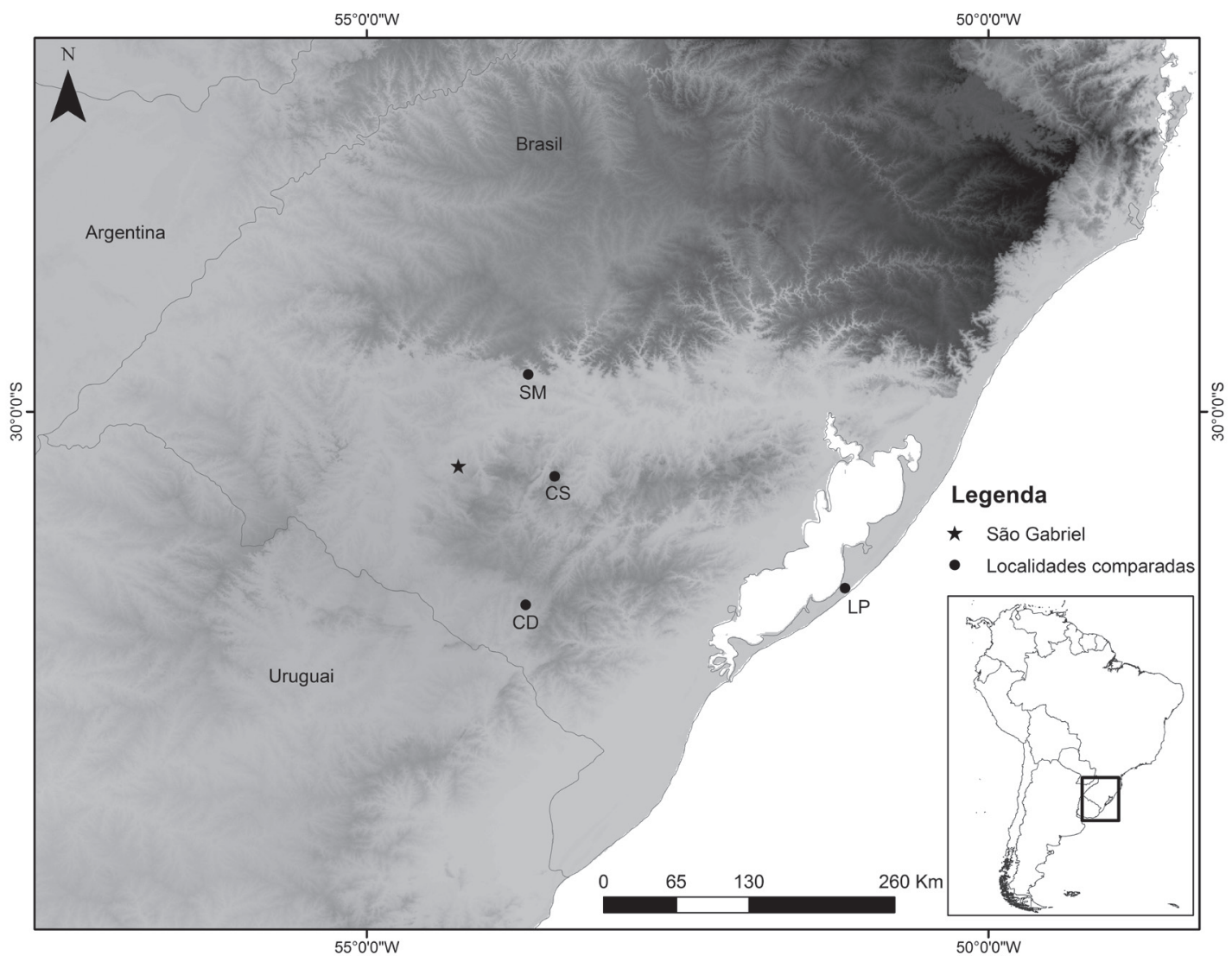

Fig. 1. Localização do município de São Gabriel, Rio Grande do Sul, Brasil, e das localidades comparadas quanto a composição de espécies de anuros na análise de similaridade. CS: Caçapava do Sul; CD: Candiota; LP: Parque Nacional da Lagoa do Peixe; SM: Santa Maria. Mapa: Carolina Pietczak. 
interior de bromélias e tocas. Exemplares testemunhos foram coletados e depositados na Coleção Científica do Setor de Zoologia do Departamento de Biologia da Universidade Federal de Santa Maria (ZUFSM; licença SISBIO/IBAMA 14596-1).

Os dados mensais de temperatura média e precipitação pluviométrica acumulada foram obtidos nos Instituto Nacional de Meteorologia, referente à estação meteorológica de São Gabriel, distante 4,5 km da área de estudo. Os modos reprodutivos foram classificados de acordo com HADDAD \& PRADO (2005), seguindo literatura disponível e observações em campo (casais em amplexo, desovas e girinos).

Análises estatísticas. A curva de acumulação de espécies foi calculada a partir de 500 adições aleatórias das amostras, utilizando o programa EstimateS 8.0 (Colwell, 2004). Adicionalmente, estimou-se a riqueza com base no uso do estimador Jacknife 2. Duas espécies de anuros identificadas tardiamente [Scinax nasicus (Cope, 1862) e Leptodactylus chaquensis Cei, 1950] não foram incluídas nos cálculos.

A composição de espécies de anuros de São Gabriel (dados obtidos na Fepagro e em outros locais do município pela mesma equipe deste trabalho) foi comparada com quatro localidades do bioma Pampa brasileiro. Segundo MARroni (2009), o Pampa possui sete regiões fisiográficas e neste estudo, foi realizada a comparação da anurofauna em áreas compreendidas em quatro delas: Campanha, Depressão Central, Litoral e Serra do Sudeste, situadas nas localidades de Candiota, Santa Maria, Parque Nacional da Lagoa do Peixe e Caçapava do Sul, respectivamente (Fig. 1). A desigualdade no esforço amostral, tamanho da área amostrada, características e status de conservação das localidades e diferenças nos conceitos taxonômicos empregados pelos pesquisadores pode afetar a comparação entre comunidades de diferentes localidades (SANTOS et al., 2009; Iop et al., 2011). Dessa forma, tomamos algumas precauções para minimizar esses efeitos. Espécies listadas como sp., gr. (grupo de espécies), cf. (confer) ou aff. (affinis) foram excluídas e a espécie exótica Lithobates catesbeianus não foi considerada na análise.

A comparação foi realizada usando o Índice de Similaridade de Jaccard $(\mathrm{Cj})$, com posterior análise de agrupamento ("clustering") pelo método de média não ponderada (UPGMA; KREBS, 1999). A representatividade das matrizes de similaridade nos dendrogramas foi verificada pela determinação do coeficiente de correlação cofenético (r). A influência da autocorrelação espacial sobre a composição de espécies de anuros entre as localidades comparadas foi analisada através de um teste de Mantel (999 permutações).

As espécies encontradas foram classificadas segundo o índice de constância de ocorrência (DAJOZ, 2005), no qual estas foram consideradas: frequentes - presentes em mais de $50 \%$ das amostragens; espécies comuns - presentes entre 25 e 50\% das amostragens e espécies raras - presentes em menos de $25 \%$ das amostragens. Para o cálculo de constância de ocorrência, foram considerados apenas os indivíduos adultos.

\section{RESULTADOS}

Foram encontradas 21 espécies de anuros distribuídas em cinco famílias e dez gêneros (Tab. I). A riqueza estimada foi de 19,99 espécies ( $\pm 0,75$ espécies; Jackknife 2). A curva de acumulação de espécies apresenta tendência de estabilização (Fig. 2). Dentre os quatro ambientes amostrados regularmente, o Ponto 4 foi o que apresentou maior riqueza $(\mathrm{N}=18)$, seguido do Ponto 2 $(\mathrm{N}=12)$ e Pontos 1 e $3(\mathrm{~N}=11)$.

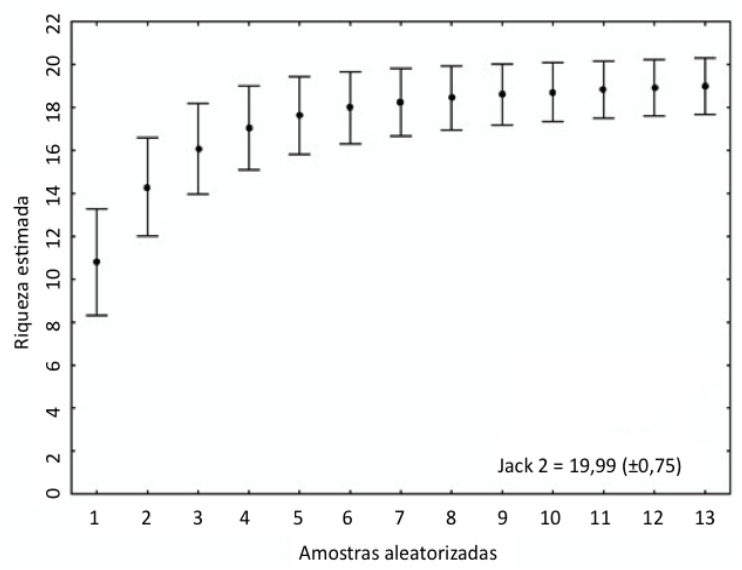

Fig. 2. Curva cumulativa para 19 espécies de anuros registradas na Fundação Estadual de Pesquisa Agropecuária, município de São Gabriel, Rio Grande do Sul, entre janeiro de 2009 e fevereiro de 2010. Os pontos expressam a curva cumulativa média, gerada por 500 adições aleatórias das amostras (meses), e as barras verticais indicam a variação possível em torno da curva média (intervalo de confiança de 95\%).

A constância de ocorrência indicou dez espécies como frequentes, sete comuns e quatro raras (Tab. I). Foram registrados três modos reprodutivos dentre as 21 espécies da área estudada (Tab. I). Doze espécies (57,15\%) utilizam o modo 1 (modo reprodutivo generalizado, caracterizado pela deposição de ovos e desenvolvimento de girinos em ambientes lênticos), cinco espécies (23,80\%) utilizam o modo 11 (modo reprodutivo caracterizado por desova em ninho de espuma depositado na superfície da água com desenvolvimento dos girinos em ambientes lênticos) e quatro espécies $(19,05 \%)$ apresentaram o modo 30 (modo reprodutivo caracterizado por desova em câmara subterrânea com desenvolvimento dos girinos em ambientes lênticos). $\mathrm{O}$ modo reprodutivo 1 foi o mais comum entre as espécies consideradas frequentes (oito dentre 10 espécies, Tab. I).

Ao comparar a anurofauna das diferentes localidades, através da análise de similaridade, observamos a formação de um grande grupo, com 55\% de similaridade, formado pelas localidades de São Gabriel, Candiota, Santa Maria e Caçapava do Sul. O Parque Nacional da Lagoa do Peixe foi a localidade que apresentou menor 
Tab. I. Famílias e espécies de anfíbios anuros registrados na Fundação Estadual de Pesquisa Agropecuária, São Gabriel, RS, no período de janeiro de 2009 a janeiro de 2010 [FO, frequência de ocorrência (\%); CO, constância de ocorrência (F, frequentes; C, comuns; R, raras); MR, modo reprodutivo; P1, Ponto 1(açude); P2, Ponto 2 (poça semi-permanente); P3, Ponto 3(poça permanente); P4, Ponto 4 (poça temporária); O, outros ambientes onde foi feita procura ativa; SG, espécies conhecidas para o município de São Gabriel].

\begin{tabular}{|c|c|c|c|c|c|c|c|c|c|}
\hline Família/espécie & FO & $\mathrm{CO}$ & MR & P1 & $\mathrm{P} 2$ & $\mathrm{P} 3$ & P4 & $\mathrm{O}$ & SG \\
\hline \multicolumn{10}{|l|}{ Bufonidae } \\
\hline Rhinella dorbignyi (Duméril \& Bibron, 1841) & - & - & 1 & & & & & & $\mathrm{x}$ \\
\hline Rhinella schneideri (Werner, 1894) & 15,38 & $\mathrm{R}$ & 1 & $\mathrm{x}$ & $\mathrm{x}$ & & & & $\mathrm{x}$ \\
\hline \multicolumn{10}{|l|}{ Hylidae } \\
\hline Dendropsophus minutus (Peters, 1872) & 53,84 & $\mathrm{~F}$ & 1 & $\mathrm{x}$ & $\mathrm{x}$ & $\mathrm{x}$ & $\mathrm{x}$ & & $\mathrm{x}$ \\
\hline Dendropsophus sanborni (Schmidt, 1944) & 69,23 & $\mathrm{~F}$ & 1 & $\mathrm{x}$ & $\mathrm{x}$ & $\mathrm{x}$ & $\mathrm{x}$ & $\mathrm{x}$ & $\mathrm{x}$ \\
\hline Hypsiboas pulchellus (Duméril \& Bibron, 1841) & 84,61 & $\mathrm{~F}$ & 1 & $\mathrm{x}$ & $\mathrm{x}$ & $\mathrm{x}$ & $\mathrm{x}$ & $\mathrm{x}$ & $\mathrm{x}$ \\
\hline Phyllomedusa iheringii Boulenger, 1885 & - & - & 24 & & & & & & $\mathrm{x}$ \\
\hline Pseudis minuta (Günther, 1858) & 92,30 & $\mathrm{~F}$ & 1 & $\mathrm{x}$ & $\mathrm{x}$ & $\mathrm{x}$ & $\mathrm{x}$ & & $\mathrm{x}$ \\
\hline Scinax fuscovarius (Lutz, 1925) & 61,53 & $\mathrm{~F}$ & 1 & & & $\mathrm{x}$ & $\mathrm{x}$ & & $\mathrm{x}$ \\
\hline Scinax granulatus (Peters, 1871) & 46,15 & $\mathrm{C}$ & 1 & & & & $\mathrm{x}$ & & $\mathrm{x}$ \\
\hline Scinax nasicus (Cope, 1862) & 30,76 & $\mathrm{C}$ & 1 & & & & $\mathrm{x}$ & & $\mathrm{x}$ \\
\hline Scinax squalirostris (Lutz, 1925) & 100 & $\mathrm{~F}$ & 1 & $\mathrm{x}$ & $\mathrm{x}$ & $\mathrm{x}$ & $\mathrm{x}$ & $\mathrm{x}$ & $\mathrm{x}$ \\
\hline \multicolumn{10}{|l|}{ Leptodactylidae } \\
\hline Leptodactylus chaquensis Cei, 1950 & 7,69 & $\mathrm{R}$ & 30 & & & & $\mathrm{x}$ & & $\mathrm{x}$ \\
\hline Leptodactylus gracilis (Duméril \& Bibron, 1840) & 46,15 & $\mathrm{C}$ & 30 & & & & $\mathrm{x}$ & $\mathrm{x}$ & $\mathrm{x}$ \\
\hline Leptodactylus latinasus (Jiménez de la Espada, 1875) & 20,07 & $\mathrm{R}$ & 30 & & & & & $\mathrm{x}$ & $\mathrm{x}$ \\
\hline Leptodactylus latrans (Steffen, 1815) & 100 & $\mathrm{~F}$ & 11 & $\mathrm{x}$ & $\mathrm{x}$ & $\mathrm{x}$ & $\mathrm{x}$ & $\mathrm{x}$ & $\mathrm{x}$ \\
\hline Leptodactylus mystacinus (Burmeister, 1861) & 7,69 & $\mathrm{R}$ & 30 & & & & & $\mathrm{x}$ & $\mathrm{x}$ \\
\hline Physalaemus biligonigerus (Cope, 1861) & 30,76 & $\mathrm{C}$ & 11 & & & & $\mathrm{x}$ & $\mathrm{x}$ & $\mathrm{x}$ \\
\hline Physalaemus cuvieri (Fitzinger, 1826) & 46,15 & $\mathrm{C}$ & 11 & & $\mathrm{x}$ & $\mathrm{x}$ & $\mathrm{x}$ & & $\mathrm{x}$ \\
\hline Physalaemus gracilis (Boulenger, 1883) & 46,15 & $\mathrm{C}$ & 11 & $\mathrm{x}$ & $\mathrm{x}$ & $\mathrm{x}$ & $\mathrm{x}$ & $\mathrm{x}$ & $\mathrm{x}$ \\
\hline Physalaemus riograndensis Milstead, 1960 & 53,84 & $\mathrm{~F}$ & 11 & $\mathrm{x}$ & & $\mathrm{x}$ & $\mathrm{x}$ & $\mathrm{x}$ & $\mathrm{x}$ \\
\hline Pseudopaludicola falcipes (Hensel, 1867) & 100 & $\mathrm{~F}$ & 1 & $\mathrm{x}$ & $\mathrm{x}$ & $\mathrm{x}$ & $\mathrm{x}$ & $\mathrm{x}$ & $\mathrm{x}$ \\
\hline \multicolumn{10}{|l|}{ Microhylidae } \\
\hline Elachistocleis bicolor (Guérin-Méneville, 1838) & 46,15 & $\mathrm{C}$ & 1 & & $\mathrm{x}$ & & $\mathrm{x}$ & $\mathrm{x}$ & $\mathrm{x}$ \\
\hline \multicolumn{10}{|l|}{ Cycloramphidae } \\
\hline Odontophrynus americanus (Duméril \& Bibron, 1841) & 61,53 & $\mathrm{~F}$ & 1 & $\mathrm{x}$ & $\mathrm{x}$ & & $\mathrm{x}$ & $\mathrm{x}$ & $\mathrm{x}$ \\
\hline Riqueza de espécies & & & & 11 & 12 & 11 & 18 & 13 & 23 \\
\hline
\end{tabular}

Tab. II. Localidades utilizadas para análise de similaridade da comunidade de anfíbios anuros do município de São Gabriel, Rio Grande do Sul, com suas respectivas regiões fisiográficas, distância $(\mathrm{Km})$ do município de São Gabriel, número de espécies e referência bibliográfica (SG, São Gabriel; CD, Candiota; CS, Caçapava do Sul; LP, Parque Nacional da Lagoa do Peixe; SM, Santa Maria).

\begin{tabular}{lcccc}
\hline Localidade & Região fisiográfica & Distância $(\mathrm{Km})$ & $\mathrm{N}^{\mathbf{o}}$ de espécies & Referência \\
\hline SG & Campanha & 0 & 23 & Presente trabalho \\
CD & Campanha & 150 & 22 & Di-BERNARDO et al., 2004 \\
CS & Serra do Sudeste & 81 & 23 & TRINDADE et al., 2010 \\
LP & Litoral & 308 & 10 & MoREIRA et al., 2010 \\
SM & Depressão Central & 87 & 25 & SANTOS et al., 2008 \\
\hline
\end{tabular}

similaridade com os demais municípios analisados (Fig. 3). O cluster teve um coeficiente de correlação cofenética $\mathrm{r}=0,97$. O teste de Mantel constatou a influência da distância sobre os agrupamentos, evidenciando que comunidades geograficamente mais próximas são mais parecidas quanto à composição da anurofauna $(\mathrm{r}=0,80$; $\mathrm{p}=0,03)$. As duas localidades com maior similaridade na composição da comunidade de anfíbios anuros são próximas geograficamente e situam-se na mesma região fisiográfica, nos municípios de São Gabriel e Candiota, ambos na Campanha Gaúcha (Tab. II, Fig 3.).

\section{DISCUSSÃO}

Estimamos a ocorrência de, pelo menos, 23 espécies de anuros para município de São Gabriel, considerando as 21 espécies deste estudo e mais duas: Phyllomedusa iheringii Boulenger, 1885 e Rhinella dorbignyi Duméril \&
Bibron, 1841 encontradas em coletas esporádicas realizadas em outras localidades do município. Esses números parecem representar, ou estar próximos, da real riqueza da área, demonstrada pela tendência de estabilização da curva de acumulação de espécies e pela comparação com outros estudos realizados na parte brasileira do bioma Pampa: 25 espécies no campus da Universidade Federal de Santa Maria, em Santa Maria (SANTos et al., 2008), 22 em Candiota (Di-Bernardo et al., 2004), 23 em Caçapava do Sul (Trindade et al., 2010) e 10 no Parque Nacional da Lagoa do Peixe, Rio Grande (Moreira et al., 2010). As 21 espécies registradas na área do estudo correspondem a cerca de 20\% das listadas para o Rio Grande do Sul (MACHADO \& Maltchiк, 2007; Rosset, 2008; Iop et al., 2009; Colombo et al., 2010; CALDART et al., 2010) e 42\% das 50 espécies citadas para a ecorregião Savana Uruguaia (GARCIA et al., 2007). São espécies características do bioma Pampa, embora não exclusivas, comumente associadas a formações 


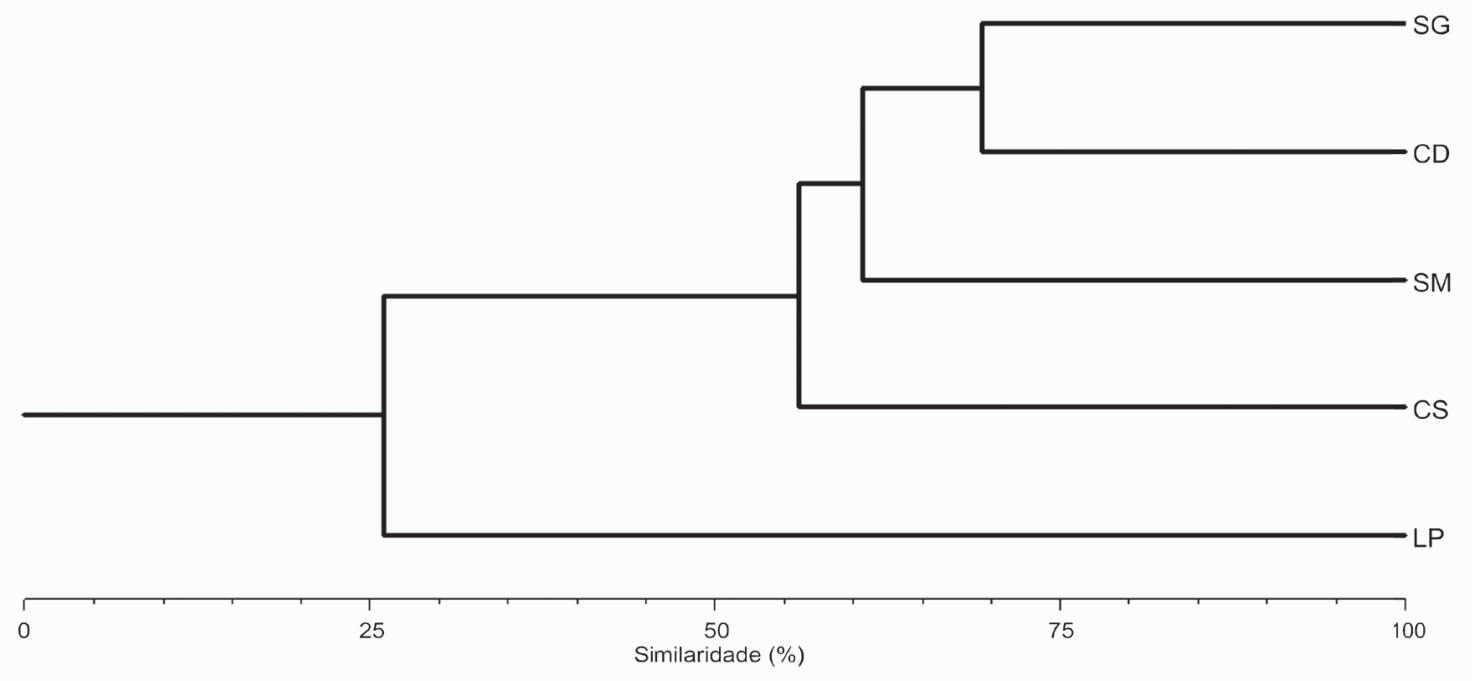

Fig. 3. Similaridade de Jaccard quanto à composição da anurofauna registrada em cinco localidades inseridas no bioma Pampa, Rio Grande do Sul. CS: Caçapava do Sul (Serra do Sudeste); CD: Candiota (Campanha); LP: Parque Nacional da Lagoa do Peixe (Litoral); SM: Santa Maria (Depressão Central); SG: São Gabriel (Campanha).

campestres do sul do Brasil e de países vizinhos, como Argentina e Uruguai (Braun \& Braun, 1980; Maneyro \& Langone, 2001; Alvarez et al., 2002; Maneyro \& CARReira, 2012).

A diferença na riqueza entre os pontos salienta a necessidade de amostrar locais com diferentes características estruturais, possibilitando estimativas de riqueza mais próximas da realidade. Os pontos 1,2 e 3 foram semelhantes no número de espécies encontradas (11,12 e 11 espécies em cada local, respectivamente) e nenhuma espécie foi exclusiva desses locais. No ponto 4 , a única poça temporária, encontramos 18 espécies, sendo quatro exclusivas: Scinax granulatus, Scinax nasicus, Leptodactylus chaquensis e Leptodactylus gracilis. Nesta poça, a vegetação era mais densa na borda, diferente dos outros pontos amostrados e sua ocupação pode estar associada com a necessidade de ambientes mais protegidos para vocalização e postura de ovos. Este também foi o ponto amostral menos utilizado pelo gado na área de estudo. Fêmeas de anuros são capazes de selecionar sítios de oviposição mais favoráveis à sobrevivência e desenvolvimento de seus ovos e girinos (Silva \& Giaretta, 2008). Desta forma, algumas espécies podem ter selecionado a poça temporária em detrimento das outras, que aparentemente eram mais impactadas pelo pisoteio do gado. No entanto, como não registramos dados sistemáticos sobre a heterogeneidade ambiental indicamos a necessidade de mais estudos sobre esse assunto no bioma Pampa.

A maioria das espécies encontradas utiliza o modo reprodutivo 1, o que concorda com SANTOS et al. (2008), que também encontraram maior proporção de espécies com modo reprodutivo generalizado em outra área do Pampa. Esses autores sugerem que a baixa diversificação e o predomínio de espécies com o modo reprodutivo 1 parecem estar mais relacionados à homogeneidade da área. Em áreas altamente heterogêneas, como da Floresta Atlântica (e. g., com presença de costões rochosos, riachos de montanha, bromélias, serapilheira úmida), apresentam maior diversidade de modos reprodutivos (HADDAD \& Prado, 2005). Ou seja, essa pequena diversificação de modos reprodutivos é esperada em ambientes não florestados, pois a diversidade de modos é considerada mais um reflexo das características ambientais do que relações filogenéticas (Duellman \& Trueb, 1994). As desovas em ninho de espuma (modos 11 e 30) também parecem estar relacionas às características ambientais (SANTOS et al., 2008). A construção de ninho de espuma pelos anuros pode servir como proteção contra dessecação dos ovos, prover temperaturas adequadas para o desenvolvimento de ovos e embriões e proteção contra radiação solar (DoBkIN \& Gettinger, 1985; Downie, 1988; Wells, 2007).

A distância geográfica, medida em quilômetros, explicou a diferença na composição das espécies. Assim, como esperado, o estudo feito no Parque Nacional da Lagoa do Peixe (Moreira et al., 2010), apresentou menor similaridade com os demais grupos. Além de ser a maior distância da área de estudo deste trabalho, a região fisiográfica desta localidade é a Planície Litorânea, sendo aceitável que seja a região fisiográfica mais diferenciada entre os municípios incluídos na análise. Duas espécies corroboram essa segregação: Odontophrynus maisuma Rosset, 2008 e Rhinella arenarum Hensel, 1867 que têm sua distribuição restrita a Planície Litorânea do estado do Rio Grande do Sul (Borges-Martins et al., 2007; Maneyro \& CARREIRA, 2012) e ocorreram somente nessa localidade.

As duas localidades com maior similaridade na composição de espécies não foram as mais próximas geograficamente, mas as únicas que tinham em comum a mesma região fisiográfica: São Gabriel (este estudo) e Candiota (Di-BERnARdo et al., 2004) pertencentes à 
Campanha Gaúcha. Dentro do grande grupo formado na análise de similaridade ainda estão Santa Maria (SAntos et al., 2008) e Caçapava do Sul, (TRINDAde et al., 2010), pertencentes à Depressão Central e Serra do Sudeste respectivamente, regiões que compartilham áreas fitoecológicas e possuem matas de encosta e matas de galeria, onde a transição para os campos não são tão abruptas como nas outras regiões. A Depressão Central é a região que possui maior contato com a Mata Atlântica e também foi a que apresentou maior número de espécies.

A riqueza total estimada para o Pampa brasileiro é de 36 espécies, somente considerando este e os outros quatro estudos realizados neste bioma (Di-BERNARDo et al., 2004; SANTOS et al., 2008; Moreira et al., 2010; TrINDADE et al., 2010). No entanto, ainda existem poucas informações a respeito dos anfíbios do bioma Pampa, em função de muitas áreas permanecerem sem inventários, evidenciando a carência de estudos envolvendo comunidades, sendo este um dos fatores limitantes para a avaliação do status de conservação deste grupo (GARCiA \& Vinciprova, 2003). Mesmo com a grande diversidade de espécies e da importância econômica, principalmente para a pecuária, os campos do bioma Pampa tem somente cerca de 3\% de sua área total legalmente protegia em unidades de conservação (MMA, 2007). A principal ameaça à conservação das espécies é a perda de habitat, causada principalmente pela rápida expansão agrícola. Neste processo, a conversão de áreas alagadas naturais, essenciais para os anuros, em áreas de cultivo, está entre as principais ameaças à conservação das características naturais deste bioma (Garcia \& Vinciprova, 2003; Bencke, 2009). Por isso, inventariar espécies nas diferentes localidades do Pampa pode fortalecer as tomadas de decisões sobre a gestão deste bioma. Além disso, o aumento de inventários faunísticos pode favorecer o conhecimento das áreas de distribuição das espécies, fornecendo, deste modo, informações mais robustas para seleção de áreas prioritárias para conservação dos anfíbios.

Agradecimentos. À Melise L. Silveira e Caroline Angri pelo auxílio e coleguismo em campo. Ao Prof. Dr. Tiago G. dos Santos pelo auxílio na identificação das espécies e sugestões no trabalho. Ao Prof. Dr. Michel V. Garey por sugestões no trabalho. Ao ICMBio pela licença concedida e ao CNPq pelo auxilio financeiro.

\section{REFERÊNCIAS BIBLIOGRÁFICAS}

Alvarez, B. B.; Aguirre, R. H.; Céspedez, J. A.; Hernando, A. B. \& Tedesco, M. E. 2002. Atlas de anfíbios y reptiles de las províncias de Corrientes, Chaco y Formosa (Argentina). Corrientes, Universidad Nacional del Nordeste. 160p.

Bencke, G. A. 2009. Diversidade e conservação da fauna dos campos do Sul do Brasil. In: Pillar, V. P.; Müller, S. C.; Castillos, Z. M. S. \& JACQues, A. V. A. eds. Campos Sulinos, conservação e uso sustentável da biodiversidade. MMA, Brasília. p.101-121.

Borges-Martins, M.; Colombo, P.; Zank, C.; Becker, F. G. \& Melo, M. T. Q. 2007. Anfibios. In: Becker, F. G.; Ramos, R. A. \& Moura, L. DE A. orgs. Biodiversidade: Regióes da Lagoa do Casamento e dos Butiazais de Tapes, Planície Costeira do Rio Grande do Sul. MMA, Brasília. p.276-291.
Braun, P. C. \& Braun, C. A. S. 1980. Lista prévia dos anfíbios do Estado do Rio Grande do Sul, Brasil. Iheringia, Série Zoologia (56):121-146.

Caldart, V. M.; Iop, S.; Santos, T. G. dos \& Cechin, S. T. Z. 2010. Extension of the geographical distribution of two anuran species for Rio Grande do Sul state, Brazil, with comments on natural history. Biota Neotropica 10(3). Disponível em: <http://www. biotaneotropica.org.br/v10n3/pt/abstract?article+bn02610032010>.

Colombo, P.; Zank, C.; Bühler, D.; Schossler, M.; Balestrin, R. L.; Santos, R. R.; Leonardi, S. B.; Borges-Martins, M. \& Verrastro, L. 2010. Anura, Hylidae, Dendropsophus nahdereri (Lutz and Bokermann, 1963): Distribution extension and new state record. Check List 6(3):429-431.

ColweLl, R. K. 2004. EstimateS 7.00: Statistical estimation of species richness and shared species from samples. Disponível em: $<\mathrm{http}: / /$ viceroy.eeb.uconn.edu/estimates>.

DAJOz, R. 2005. Princípios de Ecologia. 7ed. Porto Alegre, Artmed. 519p.

Di-Bernardo, M.; Oliveira, R. B.; Pontes, G. M. F.; Melchiors, J.; Solé, M. \& Kwet, A. 2004. Anfíbios anuros da região de extração e processamento de carvão de Candiota, RS, Brasil. In: TeIXeIrA, E. C. \& PIRES, M. J. R. eds. Estudos ambientais em Candiota: carvão e seus impactos. Porto Alegre, FEPAM. p.163-175.

Dobkin, D. S. \& GeTtingER, R. D. 1985. Thermal aspects of anuran foam nests. Journal of Herpetology 19:271-275.

Dodd JR., C. K. 2010.Amphibiam Ecology and Conservation: A Handbook of Techiniques. New York, Oxford University Press. $556 \mathrm{p}$.

DownIE, J. R. 1988. Functions of the foam in the foam-nesting leptodactylid Physalaemus pustulosus. Herpetological Journal 1:302-307.

Duellman, W. E. \& Trueb, L. 1994. Biology of Amphibians. London, The Johns Hopkins University Press. 670p.

Garcia, P. C. A. \& Vinciprova, G. 2003. Anfíbios. In: Fontana, C. S.; Bencke, G. A. \& Reis, R. E. Dos. orgs. Livro vermelho da fauna ameaçada de extinção no Rio Grande do Sul. Porto Alegre, EDIPUCRS. p.85-100.

Garcia, P. C. A.; LaVilla, E.; Langone, J. \& Segalla, M. V. 2007. Anfíbios da região subtropical da América do Sul, Padrões de distribuição. Ciência e Ambiente 35:65-100.

Haddad, C. B. \& Prado, C. A. 2005. Reproductive modes in frogs and their unexpected diversity in the Atlantic Forest of Brazil. BioScience 55(3):207-217.

Hasenack, H.; Weber, E.; Boldrini, I. I. \& Trevisan, R. 2010. Mapa de sistemas ecológicos da ecorregião das savanas uruguaias em escala 1:500.000 ou superior e relatório técnico descrevendo insumos utilizados e metodologia de elaboração do mapa de sistemas ecológicos. Porto Alegre, Universidade Federal do Rio Grande do Sul, Centro de Ecologia.

Hasenack, H.; Cordeiro, J. L. P. \& Costa, B. S. C. 2007. Cobertura vegetal atual do Rio Grande do Sul. In: DALL'AGNOL, M.; NABINGER, C.; Sant'anna, D. M. \& Santos, R. J. eds. II Simpósio de Forrageiras e Produção Animal. Porto Alegre, Departamento de Forrageiras e Agrometeorologia/UFRGS. p.15-21.

IBAMA. 2006. Parecer da equipe técnica sobre o Bioma Pampa. Disponível em: <http://br.geocities.com/biomapampa/parecer/ parecer_gt_ibama.pdf $>$. Acesso em: 29.09.2006.

Iop, S.; Caldart, V. M.; Rocha, M. C.; Paim, P. \& Ceehin, S. Z. 2009. Amphibia, Anura, Hylidae, Hypsiboas curupi Garcia, Faivovich, Haddad, 2007: First record for the state of Rio Grande do Sul, Brazil. Check List 5(4):860-862.

Iop, S.; Caldart, V. M.; Santos, T. G.; Cechin, S. Z. 2011. Anurans of Turvo State Park: testing the validity of Seasonal Forest as a new biome in Brazil. Journal of Natural History 45(39-40):2443-2461.

KreBs, C. J. 1999. Ecological methodology. Menlo Park, Addison Wesley Longman. 620p.

LidDLE, M. J. \& SCORGIE, R. A. 1980. The effects of recreation on freshwater plants and animals: A review. Biology Conservation 17:183-206.

Machado, I. F. \& Maltchiк, L. 2007. Check-list da diversidade de anuros no Rio Grande do Sul (Brasil) e proposta de classificação para as formas larvais. Neotropical Biology and Conservation 2(2):101-116.

Maneyro, R. \& Carreira, S. 2012. Guía de Anfibios del Uruguay. Montevideo, Ediciones de la fuga (Colección Ciencia Amiga). 207p. 
Maneyro, R. \& Langone, J. A. 2001. Categorización de los anfíbios del Uruguay. Cuadernos de Herpetología 15(2):107-118.

Marroni, F. 2009. Bioma Pampa. Patrimônio Nacional. Série Separatas de discursos, pareceres e projetos, $\mathrm{n}^{\circ}$.108. Brasília. Disponível em: $<$ http://issuu.com/fernandomarroniperfillotado/docs/bioma_pampa $>$. Acesso em: 03.07.2013.

MMA. 2007. Áreas Prioritárias para Conservação, Uso Sustentável e Repartição de Benefícios da Biodiversidade: Atualização-Portaria MMA nº 9 , de 23 de janeiro de 2007. Brasília, Ministério do Meio Ambiente, Secretaria de Biodiversidade e Florestas. p.87-95.

Moreira, L. F. B.; Machado, I. F.; Garcia, T. V. \& Maltchik, L. 2010. Factors influencing anuran distribution in coastal dune wetlands in southern Brazil. Journal of Natural History 44(23-24): 1493-1507.

Overback, G. E.; Müller, S. C.; Fidelis, A.; Pfadenhauer, J.; Pillar, V. D.; Blanco, C. C.; Boldrini, I. I.; Both, R. \& Forneck, E. D. 2009. Os Campos Sulinos: um bioma negligenciado. In: Pillar, V. P.; Müller, S. C.; Castilhos, Z. M. S. \& Jacques, A. V. A. eds. Campos Sulinos, conservação e uso sustentável da biodiversidade. Brasília, MMA. p.26-41.

Pereira, P. R. B.; Netto, L. R. G.; Borin, C. J. A. \& Sartori, M. G. B. 1989. Contribuição à geografia física do município de Santa Maria: unidades de paisagem. Geografia - Ensino \& Pesquisa 3:37-68.

Rosset, S. D. 2008. New Species of Odontophrynus Reinhardt and Lütken 1862 (Anura: Neobatrachia) from Brazil and Uruguay. Journal of Herpetology 42(1):134-144.

Santos, T. G.; Kopp, K.; Spies, M. R.; Trevisan, R. \& Cechin, S. Z. 2008. Distribuição temporal e espacial de anuros em área de Pampa, Santa Maria, RS. Iheringia, Série Zoologia 98(2):244-253.

Santos, T. G.; Vasconcelos, T. S.; Rossa-Feres, D. C. \& Haddad, C. F. B. 2009. Anurans of a seasonally dry tropical forest: Morro do Diabo State Park, São Paulo state, Brazil. Journal of Natural History 43(13-16):973-993.
Scott JR., N. J. \& Woodward, B. D. 1994. Surveys at breeding sites. In: Heyer, W. R.; Donnelly, M. A.; McDiarmid, R. W.; HayeK, L. A. C. \& Foster, M. S. eds. Measuring and Monitoring Biological Diversity - Standard Methods for Amphibians. Washington and London, Smithsonian Institution Press. p.84-92.

Silva, M. D. 2012. Bioma pampa, um sistema ameaçado. Revista Floresta 42(1):215-226.

Silva, W. R. \& Giaretta, A. A. 2008. Oviposition site selection in anurans (Lissamphibia). Biota Neotropica 8(2). Disponível em $<$ http://www.biotaneotropica.org.br/v8n3/pt/abstract?thematicreview+bn02008032008>.

Suertegaray, D. M. A. \& Silva, L. A. P. 2009. Tchê Pampa: histórias da natureza gaúcha. In: Pillar, V. P.; Müller, S. C.; Castilhos, Z. M. S. \& JACQues, A. V. A. eds. Campos Sulinos, conservação e uso sustentável da biodiversidade. Brasília, MMA. p.42-59.

Tocher, M. D.; Gascon, C. \& Zimmerman, B. L. 1997. Fragmentation effects on a Central Amazonian frog community: a ten-year study. In: Laurance, W. F. \& Bierregaard, R. O. eds. Tropical forest remmants: ecology, management and conservation of fragmented communities. Chicago, The University of Chicago Press. p.124-127.

Trindade, A. O.; Oliveira, S. V. \& Cappellari, L. H. 2010. Anfíbios anuros de uma área da Serra do Sudeste, Rio Grande do Sul (Caçapava do Sul). Biodiversidade Pampeana 8(1):19-24.

VAn Rooy, P. T. J. C. \& Stumpel, A. H. P. 1995. Ecological impact of economic development on sardinian herpetofauna. Conservation Biology 9:263-269.

WeLLS, K. D. 2007. The ecology and behavior of amphibians. Chicago, The University of Chicago Press. 1400p. 